\title{
Associations between serum concentrations of perfluoroalkyl acids and serum lipid levels in a Chinese population
}

\author{
Yaning $\mathrm{Fu}^{\mathrm{a}, \mathrm{b}}$, Tieyu Wang ${ }^{\mathrm{a}}$, Quanliang $\mathrm{Fu}^{\mathrm{c}}$, Pei Wang ${ }^{\mathrm{a}, \mathrm{b}}$, Yonglong $\mathrm{Lu}^{\mathrm{a}, *}$ \\ a State Key Lab of Urban and Regional Ecology, Research Center for Eco-Environmental Sciences, Chinese Academy of Sciences, Beijing 100085, China \\ ${ }^{\mathrm{b}}$ University of Chinese Academy of Sciences, Beijing 100049, China \\ ${ }^{\mathrm{c}}$ Red Cross Hospital of Yuanyang, Yuanyang County, Henan 453500, China
}

\section{A R T I C L E I N F O}

\section{Article history:}

Received 9 January 2014

Received in revised form

25 April 2014

Accepted 26 April 2014

Available online 23 May 2014

Keywords:

Perfluoroalkyl acids

Serum lipid

Cholesterol

Human health

Chinese population

\begin{abstract}
A B S T R A C T
Perfluoroalkyl acids (PFAAs) have been used in a variety of products for many years and have been detected worldwide in human serum. Previous studies have suggested the potential effects of PFAAs on serum lipids. To investigate the associations between serum concentrations of PFAAs and serum lipid levels, 133 participants were randomly selected from the people coming for health check-up in Yuanyang Red Cross Hospital of Henan, China. Linear regression analysis revealed that perfluoro-octanoic acid (PFOA), perfluorononanoic acid (PFNA), and perfluorodecanoic acid (PFDA), with a median concentration of $1.43,0.37$, and $0.19 \mathrm{ng} / \mathrm{mL}$, respectively, were positively associated with total cholesterol (TC). Those in the highest quartile of PFOA exposure had ln-TC levels $0.24 \mathrm{mmol} / \mathrm{L}$ higher than those in the lowest quartile. For PFNA and PFDA, effect estimates were 0.25 and $0.16 \mathrm{mmol} / \mathrm{L}$, respectively. A positive association between high-density lipoprotein cholesterol (HDLC) and PFDA was found, and there was a $0.18 \mathrm{mmol} / \mathrm{L}$ increase of HDLC for the top PFDA quartile compared with the lowest quartile. PFOA and PFNA were positively associated with low-density lipoprotein cholesterol (LDLC). Ln-LDLC levels of people in both top PFOA and PFNA quartiles were $0.33 \mathrm{mmol} / \mathrm{L}$ higher than those in the lowest quartiles. Logistic regression analysis indicated that increased PFOA and PFOS quartiles were positively associated with an increased risk of abnormal TC and LDLC when controlling for no confounding factors.
\end{abstract}

(c) 2014 Elsevier Inc. All rights reserved.

\section{Introduction}

As a class of synthetic chemicals with surface-active properties, perfluoroalkyl acids (PFAAs) have been widely used as surfactants and surface protectors for over 50 years in many applications, including lubricants, paints, polishes, fire-fighting foams, food packaging and so on (Lau et al., 2007). In recent years, PFAAs have attracted great attention internationally due to their global occurrences in environment and biota (Giesy and Kannan, 2001; Meesters and Schroder, 2004), as well as their toxicity (Lau et al., 2007) and bioaccumulation (Martin et al., 2004). PFAAs have also been found in potential human exposure pathways, such as food (Tittlemier et al., 2006), drinking water (Skutlarek et al., 2006), indoor air and dust (Shoeib et al., 2005), which hence raise great public-health concerns.

PFAAs have been detected from human blood or serum samples in general population from many countries (Kannan et al., 2004; Fromme et al., 2009), including China (So et al., 2006; Pan et al.,

\footnotetext{
* Corresponding author. Fax: +86 1062918177.

E-mail address: yllu@rcees.ac.cn (Y. Lu).
}

2010). PFAAs are slowly eliminated in the human body (Lau et al., 2007). Unlike many other persistent organic pollutants accumulating in lipids, PFAAs bind to proteins in the liver and serum (Conder et al., 2008). The arithmetic mean serum half-lives of perfluoro-octane sulfonate (PFOS) and perfluoro-octanoic acid (PFOA) in humans are estimated to be 5.4 years [95\% confidence interval $(\mathrm{CI}), 3.9-6.9$ years] and 3.8 years (95\% CI, 3.1-4.4 years), respectively (Olsen et al., 2007). Although the half-lives for perfluorononanoic acid (PFNA), perfluorodecanoic acid (PFDA) and perfluoroundecanoic acid (PFUdA) in humans have not been estimated, longer-chain compounds are generally assumed to have longer half-lives, which has been proven by animal studies (Martin et al., 2003).

Previous toxicological studies have shown that PFAAs have various adverse health effects, including the potential to affect circulating concentrations of cholesterol (Olsen et al., 2009; DeWitt et al., 2012; Lin et al., 2013; Seacat et al., 2002). On daily exposure to PFOS at doses of $0.75 \mathrm{mg} / \mathrm{kg}$ body weight/day over a six-month period, cynomolgus monkeys exhibited a marked decrease in serum TC at serum PFOS concentrations approximately $150,000 \mathrm{ng} / \mathrm{mL}$ (Seacat et al., 2002). Moreover, animal studies have revealed that PFAAs bind to peroxisome proliferator-activated 
receptors (PPARs), constitutive androstane receptor (CAR), pregnane $X$ receptor (PXR), and liver $X$ receptor (LXR) (Elcombe et al., 2010; Bjork et al., 2011; Elcombe et al., 2012). Nuclear receptors play a key role in lipid metabolism and adipogenesis, raising the concern that PFAAs may disrupt lipid regulation (Maher et al., 2008; Fang et al., 2012). In addition, another study utilizing the APOE*3-Leiden.CETP mouse model showed that PFOS may reduce TC by impairing lipoprotein production (Bijland et al., 2011). Compared with the effects found in animals, studies in humans have reported inconsistent associations between PFAAs and serum lipids (Frisbee et al., 2010a; Nelson et al., 2010; Fisher et al., 2013). Nelson et al. (2010) investigated four types of PFAAs and reported that people in the highest PFOA quartile had TC levels $0.25 \mathrm{mmol} / \mathrm{L}$ higher than those in the lowest quartile, while Fisher et al. (2013) found no significant association between cholesterol levels and PFOS or PFOA levels. One study on American children and adolescents, Frisbee et al. (2010a) found that between the first and fifth quintiles of PFOA, there was a 0.12 and $0.10 \mathrm{mmol} / \mathrm{L}$ increase of TC and LDLC, respectively. In a longitudinal assessment of workers involved in the demolition and disposal of perfluoroalkyl manufacturing plants, Olsen et al. (2012) observed no adverse associations between changes in PFOA, PFOS, and serum lipids. In another longitudinal study, although it was questioned by Burstyn (2013) for its clinical relevancy, Fitz-Simon et al. (2013) found that there was a tendency for people with greater declines in serum PFOA or PFOS to have greater LDLC decrease. For a person whose serum PFOA or PFOS fell by half, the predicted fall in LDLC was $3.6 \%$ or $5 \%$, respectively

The results of previous epidemiological studies are not consistent. Most of these studies have focused on PFOS and PFOA, and the influence of other PFAAs on serum lipids is seldom discussed. Most previous studies have been performed in populations of European ancestry, and few are reported in the Chinese population. In the present study, we aimed to investigate potential associations between serum concentrations of eight PFAAs and four serum lipid levels in a Chinese population.

\section{Materials and methods}

\subsection{Study population}

All of the participants in this study were recruited from the Yuanyang County of Henan Province, China. Henan, with a population of over 94 million, is the largest provincial economy among inland provinces of China. From October-November 2011, 133 participants aged from 0 to 88 years old were randomly selected from the people coming for health check-up in Yuanyang Red Cross Hospital. In the meantime, serum samples were collected from the participants. Briefly, the serum was immediately separated from approximate $5 \mathrm{~mL}$ whole blood and then stored at $-20^{\circ} \mathrm{C}$ prior to further analysis. In addition, telephone interviews were also performed to collect detailed information of each participant, including lifestyle and demographic factors. However, no information on cholesterol lowering medication was obtained. The study was approved by the Red Cross Hospital from Yuangyang County and written informed consent was obtained from each participant.

\subsection{Analysis of serum lipids}

TC, triglyceride (TG), high-density lipoprotein cholesterol (HDLC) and LDLC are commonly used in clinical and epidemiologic studies. Concentrations of TC, TG, HDLC and LDLC in sera of all participants were directly determined using a BS-800 Chemistry Analyzer (Mindray, Shenzhen, China) at the clinical laboratory of the Yuanyang Red Cross Hospital.

\subsection{Chemical analysis}

A total of 12 PFAAs were analyzed in serum samples, including perfluorobutane sulfonate (PFBS), perfluorohexane sulfonate (PFHxS), PFOS, perfluorobutyric acid (PFBA), perfluoropentanoic acid (PFPeA), perfluorohexanoic acid (PFHxA), perfluoroheptanoic acid (PFHpA), PFOA, PFNA, PFDA, PFUdA and perfluorododecanoic acid (PFDoA).

PFAAs were extracted from serum with methyl tert-butyl ether in forming an ion pair with tetrabutylammonium hydrogen sulfate. Extracts were evaporated to dryness under a stream of high-purity nitrogen, dissolved in $1 \mathrm{~mL}$ of mobile phase, and then analyzed using an Agilent 1290 high performance liquid chromatography system (Palo Alto, CA, USA) coupled with an Agilent 6460 Triple Quadrupole mass spectrometer (Santa Clara, CA, USA). The analysis was operated in multiple reaction monitoring (MRM) mode with the electrospray ionization (ESA) in negative mode. Detailed methods are provided in supplementary materials.

\subsection{Quality assurance and quality control}

To check the contamination occurred during the extraction process, procedural blanks were prepared at an interval of every 10 samples. Moreover, background contamination was monitored using solvent blanks containing acetonitrile and Milli-Q water (2:3, v/v) for every 20 samples. In addition, duplicate injections and calibration check standards were performed for every 20 samples to assure the precision and accuracy of each run. Concentrations of serum extracts were determined via nine-point matrix-matched calibration curves ranging from 0.01 to $100 \mathrm{ng} / \mathrm{mL}$, which were performed by adding mixed PFAA standard solutions into blank newborn bovine serum. The regression coefficients $\left(r^{2}\right)$ of calibration curves for all the target analytes were higher than 0.99. The limit of detection (LOD) and the limit of quantification (LOQ) were defined as three-fold and ten-fold greater than the signal-to-noise ratio, respectively. The recovery and reproducibility of the serum sample extraction were determined on six replicate analyses of $0.5 \mathrm{~mL}$ of newborn bovine serum containing $2 \mathrm{ng}$ of each PFAA standard. No PFAA contamination was found above the LOD in newborn bovine serum. Table S1 summarizes the LOD, LOQ and matrix spike recoveries for all the target chemicals.

\subsection{Statistical analysis}

Statistical analyses were conducted using SPSS Statistics v19.0 (SPSS Inc., Chicago, IL). PFPeA, PFDoA, PFBS and PFHxS were detected in less than $75 \%$ of samples $(63.91 \%, 34.59 \%, 30.08 \%$ and $26.32 \%$, respectively). Therefore, results for PFPeA, PFDoA, PFBS and PFHxS were not further discussed in our study. Concentrations of PFAAs were expressed as median or mean \pm standard deviation. Value below the LOD was assigned as a proxy value of an LOD/2

TC, TG, HDLC and LDLC were used as the outcome variables in linear regression analyses. TC, TG and LDLC were In-transformed to satisfy the normality criteria. Exposure was modeled in quartiles of PFAA concentrations. Each quartile was compared with the reference group (the first quartile). The effect estimates and the corresponding 95\% confidence intervals (CIs) were presented. PFAA category was considered as a linear predictor in the quartile analyses.

Binary logistic regression analyses were conducted to assess the odds ratio of abnormal lipids with increasing PFAA quartile. The serum lipid categorization for adults was based on the Guidelines on Prevention and Treatment of Blood Lipid Abnormality in Chinese Adults (Zhao, 2008), while its categorization for children was based on the Expert Consensus for Prevention and Treatment of Dyslipidemia in Children and Adolescents (Xiang and Du, 2009). For TC, values higher than $5.18 \mathrm{mmol} / \mathrm{L}$ in adults or $4.40 \mathrm{mmol} / \mathrm{L}$ in children were classified as abnormal. For TG, values higher than $1.70 \mathrm{mmol} / \mathrm{L}$ were classified as abnormal. For HDLC, values lower than $1.04 \mathrm{mmol} / \mathrm{L}$ were classified as abnormal. For LDLC, values higher than $3.37 \mathrm{mmol} / \mathrm{L}$ in adults or $2.85 \mathrm{mmol} / \mathrm{L}$ in children were classified as abnormal. Logistic regression analyses were performed using PFAA quartile dummy variables, in which the first quartile was considered as the reference group. Age, gender and body mass index (BMI) were included in adjusted models as covariates to assess their confounding influence.

\section{Results}

Table 1 lists general characteristics of participants in the present study. The participants consisted of 80 males and 53 females. The median age was 30 years, ranging from 0.3 to 80 years. The BMI ranged from 15.3 to 35.6, with a median value of 23.5 .

Among the 12 analyzed PFAAs, eight compounds were detected in more than $75 \%$ of the samples $(78.20 \%$ for PFBA, $76.69 \%$ for PFHxA, 98.5\% for PFHpA, 100\% for PFOA, 97.74\% for PFNA, 82.71\% for PFDA, $81.20 \%$ for PFUdA and $100.00 \%$ for PFOS). PFOS was detected with the greatest median concentration $(1.47 \mathrm{ng} / \mathrm{mL}$, range: $0.08-10.20 \mathrm{ng} / \mathrm{mL})$, followed by PFOA $(1.43 \mathrm{ng} / \mathrm{mL}$, range: $0.32-39.46 \mathrm{ng} / \mathrm{mL})$ and PFNA $(0.37 \mathrm{ng} / \mathrm{mL}$, range: $0.02-4.18$ $\mathrm{ng} / \mathrm{mL}$ ), and they accounted for about $80 \%$ of the total PFAAs. Significant correlations were detected between PFBA and PFOS, 
Table 1

Characteristics of participants.

\begin{tabular}{|c|c|c|c|c|c|}
\hline Characteristic & No. & Percentage (\%) & Median & Mean \pm SD & Range \\
\hline Age & 133 & & 30.0 & $34.0 \pm 23.0$ & $0.3-88.0$ \\
\hline Gender & 133 & & & & \\
\hline Male & 80 & 60.2 & & & \\
\hline Female & 53 & 39.8 & & & \\
\hline BMI & 133 & & 23.5 & $23.3 \pm 4.1$ & $15.3-35.6$ \\
\hline $\mathrm{TC}(\mathrm{mmol} / \mathrm{L})$ & 133 & & 4.09 & $4.41 \pm 1.29$ & $2.39-8.37$ \\
\hline Normal & 94 & 70.7 & 3.70 & $3.72 \pm 0.72$ & $2.39-5.10$ \\
\hline Abnormal & 39 & 29.3 & 5.86 & $6.04 \pm 0.79$ & $4.61-8.37$ \\
\hline TG $(\mathrm{mmol} / \mathrm{L})$ & 133 & & 1.74 & $2.00 \pm 1.15$ & $0.60-6.96$ \\
\hline Normal & 66 & 49.6 & 1.29 & $1.23 \pm 0.28$ & $0.60-1.67$ \\
\hline Abnormal & 67 & 50.4 & 2.25 & $2.75 \pm 1.18$ & $1.74-6.96$ \\
\hline HDLC (mmol/L) & 133 & & 1.32 & $1.34 \pm 0.32$ & $0.25-2.57$ \\
\hline Normal & 118 & 88.7 & 1.36 & $1.41 \pm 0.27$ & $1.06-2.57$ \\
\hline Abnormal & 15 & 11.3 & 0.94 & $0.83 \pm 0.25$ & $0.25-1.04$ \\
\hline LDLC (mmol/L) & 133 & & 2.65 & $2.96 \pm 1.23$ & $1.32-8.52$ \\
\hline Normal & 86 & 64.7 & 2.15 & $2.23 \pm 0.51$ & $1.32-3.29$ \\
\hline Abnormal & 47 & 35.3 & 4.15 & $4.31 \pm 0.98$ & $3.09-8.52$ \\
\hline PFBA (ng/mL) & 133 & & 0.11 & $0.15 \pm 0.14$ & $0.01-0.65$ \\
\hline Quartile 1 & 33 & 24.8 & 0.01 & $0.01 \pm 0.003$ & $0.01-0.02$ \\
\hline Quartile 2 & 32 & 24.1 & 0.07 & $0.07 \pm 0.02$ & $0.03-0.11$ \\
\hline Quartile 3 & 35 & 26.3 & 0.17 & $0.17 \pm 0.04$ & $0.11-0.23$ \\
\hline Quartile 4 & 33 & 24.8 & 0.33 & $0.35 \pm 0.11$ & $0.23-0.65$ \\
\hline PFHxA (ng/mL) & 133 & & 0.03 & $0.04 \pm 0.04$ & $0.01-0.32$ \\
\hline Quartile 1 & 39 & 29.3 & 0.01 & $0.01 \pm 0.004$ & $0.01-0.02$ \\
\hline Quartile 2 & 28 & 21.1 & 0.03 & $0.03 \pm 0.003$ & $0.02-0.03$ \\
\hline Quartile 3 & 33 & 24.8 & 0.04 & $0.04 \pm 0.007$ & $0.03-0.05$ \\
\hline Quartile 4 & 33 & 24.8 & 0.08 & $0.09 \pm 0.05$ & $0.05-0.32$ \\
\hline PFHpA (ng/mL) & 133 & & 0.04 & $0.05 \pm 0.04$ & $0.01-0.29$ \\
\hline Quartile 1 & 34 & 25.6 & 0.03 & $0.03 \pm 0.005$ & $0.01-0.03$ \\
\hline Quartile 2 & 32 & 24.1 & 0.04 & $0.04 \pm 0.003$ & $0.03-0.04$ \\
\hline Quartile 3 & 34 & 25.6 & 0.05 & $0.05 \pm 0.005$ & $0.04-0.06$ \\
\hline Quartile 4 & 33 & 24.8 & 0.07 & $0.08 \pm 0.04$ & $0.06-0.29$ \\
\hline PFOA (ng/mL) & 133 & & 1.43 & $2.95 \pm 4.65$ & $0.32-39.46$ \\
\hline Quartile 1 & 33 & 24.8 & 0.71 & $0.72 \pm 0.17$ & $0.32-0.99$ \\
\hline Quartile 2 & 34 & 25.6 & 1.24 & $1.23 \pm 0.12$ & $1.02-1.43$ \\
\hline Quartile 3 & 33 & 24.8 & 1.97 & $2.03 \pm 0.47$ & $1.44-2.85$ \\
\hline Quartile 4 & 33 & 24.8 & 4.88 & $7.89 \pm 7.40$ & $2.95-39.46$ \\
\hline PFNA (ng/mL) & 133 & & 0.37 & $0.56 \pm 0.62$ & $0.02-4.68$ \\
\hline Quartile 1 & 33 & 24.8 & 0.14 & $0.13 \pm 0.06$ & $0.02-0.21$ \\
\hline Quartile 2 & 34 & 25.6 & 0.30 & $0.30 \pm 0.05$ & $0.23-0.37$ \\
\hline Quartile 3 & 33 & 24.8 & 0.45 & $0.47 \pm 0.07$ & $0.37-0.63$ \\
\hline Quartile 4 & 33 & 24.8 & 1.02 & $1.32 \pm 0.83$ & $0.67-4.68$ \\
\hline PFDA (ng/mL) & 133 & & 0.19 & $0.30 \pm 0.37$ & $0.02-2.16$ \\
\hline Quartile 1 & 33 & 24.8 & 0.02 & $0.03 \pm 0.02$ & $0.02-0.07$ \\
\hline Quartile 2 & 33 & 24.8 & 0.14 & $0.13 \pm 0.03$ & $0.07-0.18$ \\
\hline Quartile 3 & 34 & 25.6 & 0.26 & $0.28 \pm 0.07$ & $0.19-0.42$ \\
\hline Quartile 4 & 33 & 24.8 & 0.62 & $0.78 \pm 0.45$ & $0.43-2.16$ \\
\hline PFUdA (ng/mL) & 133 & & 0.26 & $0.34 \pm 0.36$ & $0.02-1.93$ \\
\hline Quartile 1 & 33 & 24.8 & 0.02 & $0.03 \pm 0.02$ & $0.02-0.09$ \\
\hline Quartile 2 & 34 & 25.6 & 0.16 & $0.17 \pm 0.05$ & $0.09-0.26$ \\
\hline Quartile 3 & 33 & 24.8 & 0.35 & $0.34 \pm 0.05$ & $0.27-0.43$ \\
\hline Quartile 4 & 33 & 24.8 & 0.68 & $0.84 \pm 0.38$ & $0.51-1.93$ \\
\hline PFOS (ng/mL) & 133 & & 1.47 & $1.68 \pm 1.20$ & $0.08-10.20$ \\
\hline Quartile 1 & 33 & 24.8 & 0.62 & $0.61 \pm 0.24$ & $0.08-0.94$ \\
\hline Quartile 2 & 34 & 25.6 & 1.26 & $1.26 \pm 0.15$ & $0.96-1.47$ \\
\hline Quartile 3 & 33 & 24.8 & 1.68 & $1.76 \pm 0.20$ & $1.49-2.14$ \\
\hline Quartile 4 & 33 & 24.8 & 2.55 & $3.12 \pm 1.52$ & $2.15-10.20$ \\
\hline
\end{tabular}

PFHPA and PFNA, PFHpA and PFOS, PFOA and PFOS, PFOA and PFNA, PFOA and PFDA, PFNA and PFDA, PFDA and PFUdA, respectively (See Supplementary Fig. S1). Moreover, we also showed that concentrations of PFOA $(r=0.239, p<0.01)$, PFNA $(r=0.185$, $p<0.05)$ and PFOS $(r=0.175, p<0.05)$ were significantly associated with age, whereas no significant association was found between age and other PFAAs. Median concentrations of PFOA, PFNA, PFDA and PFOS appeared to be higher in males than those in females across all age groups.

Serum lipid levels varied with age, gender and BMI, and they were correlated with each other. Median TC was $4.09 \mathrm{mmol} / \mathrm{L}$, and $29.3 \%$ of values were classified as abnormal. Median TG was $1.74 \mathrm{mmol} / \mathrm{L}$, and $50.4 \%$ of values were classified as abnormal.
Median HDLC was $1.32 \mathrm{mmol} / \mathrm{L}$, and $11.3 \%$ of values were classified as abnormal. Median LDLC was $2.65 \mathrm{mmol} / \mathrm{L}$, and $35.3 \%$ of values were classified as abnormal.

Regression analysis (see Supplementary Table S2) demonstrated that TC was positively associated with PFOA $(p<0.001)$, PFNA $(p<0.001)$, PFUdA $(p<0.05)$, PFDA $(p<0.05)$ and PFOS $(p<0.01)$ in crude models, and it was positively associated with PFOA $(p<0.05)$, PFNA $(p<0.01)$ and PFDA $(p<0.05)$ after the information of age, gender and BMI was adjusted. TG was not associated with any PFAAs either in crude or in adjusted models. HDLC was positively associated with PFDA in both crude and adjusted models ( $p<0.05$ and $p<0.01$, respectively). LDLC was associated with PFOA $(p<0.001)$, PFNA $(p<0.01)$, PFUdA $(p<0.05)$ and PFOS $(p<0.01)$ in crude models, while it was only associated with PFOA $(p<0.05)$ and PFNA $(p<0.01)$ in adjusted models.

Fig. 1 shows the adjusted associations between increasing PFAA quartiles and the change in serum lipid levels. PFBA, PFHxA and PFHpA were excluded from Fig. 1 since they were not associated with any serum lipids across all models.

Fig. 1(A) describes a positive association between TC and concentrations of PFOA, PFNA and PFDA. Data showed that $\ln$ TC levels of people in the highest PFOA quartile were $0.24 \mathrm{mmol} / \mathrm{L}$ higher than those in the lowest quartile. For PFNA, there was a $0.25 \mathrm{mmol} / \mathrm{L}$ increase, and there was a $0.16 \mathrm{mmol} / \mathrm{L}$ increase for PFDA. TC appeared to linearly increase across quartile of PFNA $(p<0.05)$. TC also showed an increase with PFUdA and PFOS quartiles, however, the association was not significant. Fig. 1 (B) shows that no meaningful associations were observed between TG and PFAAs. A positive association between HDLC and PFDA was found, and there was a $0.18 \mathrm{mmol} / \mathrm{L}$ increase of HDLC for the top PFDA quartile compared with the lowest quartile (Fig. 1(C)). A positive association between LDLC and concentrations of PFOA and PFNA was found (Fig. 1(D)). In addition, In LDLC levels of people in both top PFOA and PFNA quartiles were $0.33 \mathrm{mmol} / \mathrm{L}$ higher than those in their lowest quartiles.

Table 2 shows logistic regression results. The increase of PFOA and PFOS quartiles were positively associated with an increased risk of abnormal TC and LDLC in crude models. However, this association became insignificant in adjusted models. No other significant association was found between other PFAAs and serum lipids either in crude or in adjusted models.

\section{Discussion}

To our best knowledge, we, for the first time, investigated associations between PFAA concentrations and serum lipids in China and linked PFDA concentrations with serum lipids.

In our present study, median concentrations of PFAAs were lower than those previously reported from other parts of China, and the composition profile of PFAAs was quite different. PFOA, instead of PFOS, was the dominant analyte (Pan et al., 2010; Guo et al., 2011). Consistent with other studies, the univariate analysis showed that concentrations of PFOA, PFNA and PFOS were significantly correlated with age, and concentrations of PFOA, PFNA, PFDA and PFOS appeared to be higher in males than those in females (Calafat et al., 2007; Fromme et al., 2007; Holzer et al., 2008; Haug et al., 2009; Zhang et al., 2010).

As shown in Supplementary Table S3, previous studies have investigated the associations between serum lipids and PFAAs concentrations (Emmett et al., 2006; Olsen and Zobel, 2007; Sakr et al., 2007a; Sakr et al., 2007b; Frisbee et al., 2010a; Nelson et al., 2010; Eriksen et al., 2013; Fisher et al., 2013). However, those data are not always consistent. One cross-sectional study carried out among occupational population with a much higher exposure 


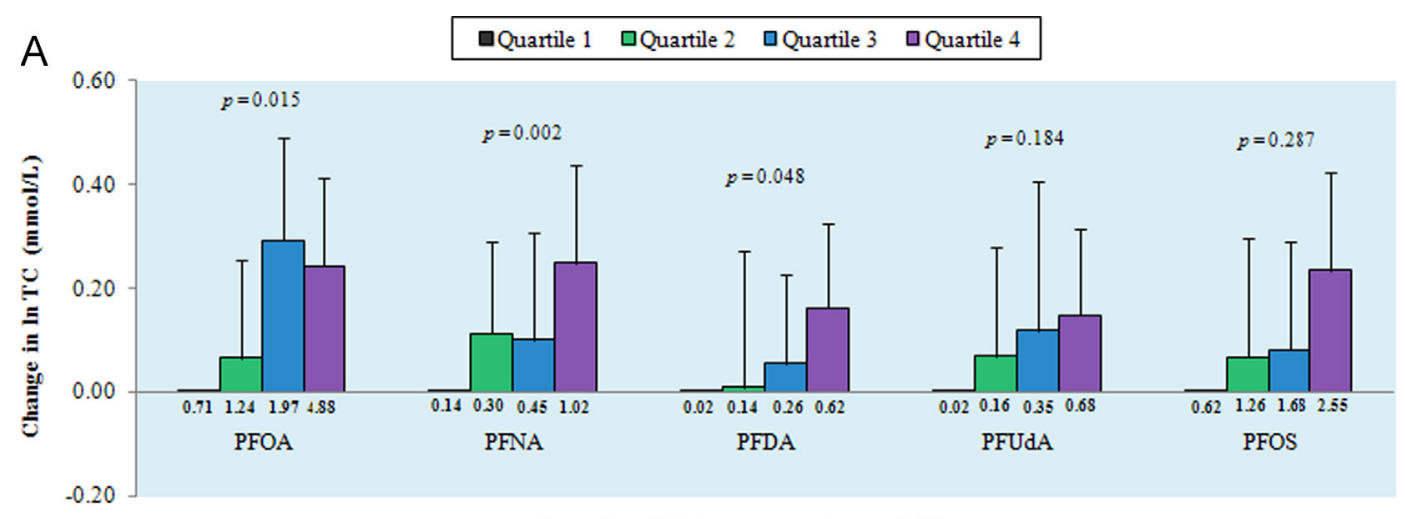

Quartile of PFAAs concentrations (ng/mL)

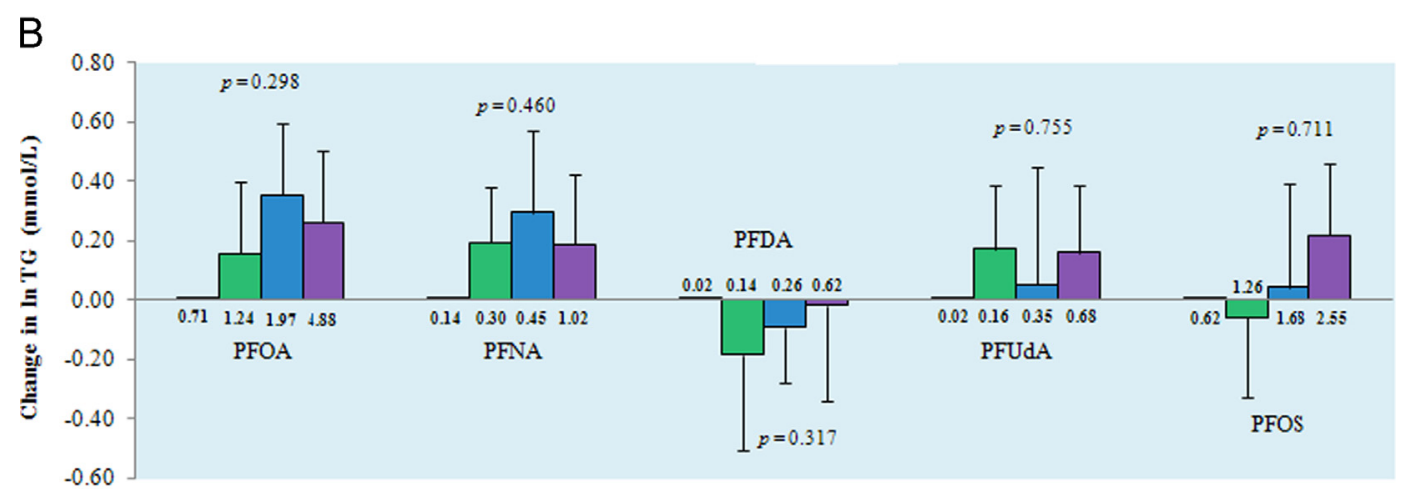

Quartile of PFAAs concentrations ( $\mathrm{ng} / \mathrm{mL}$ )

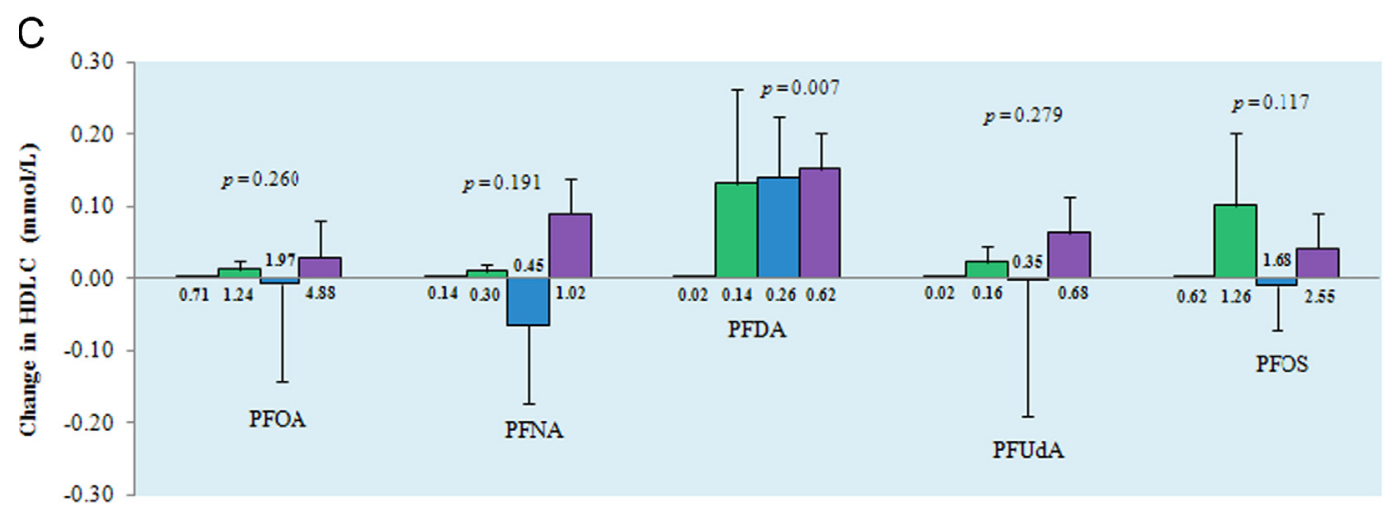

Quartile of PFAAs concentrations (ng/mL)

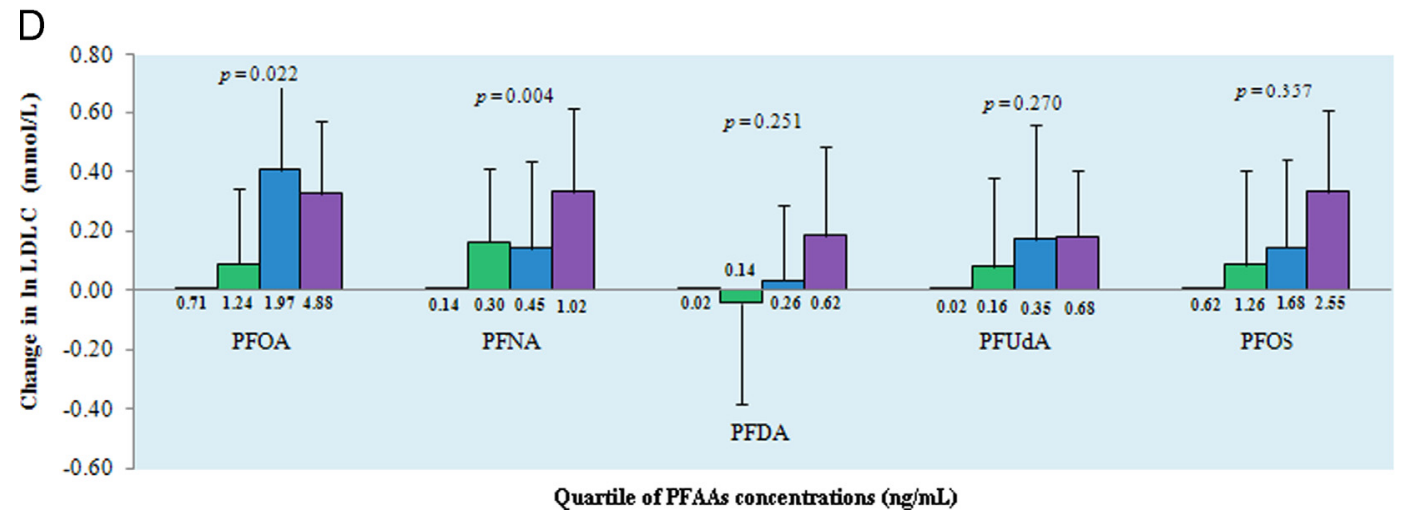

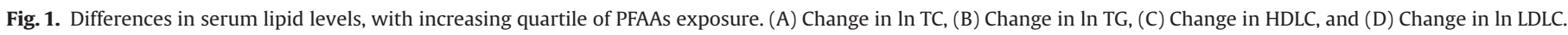

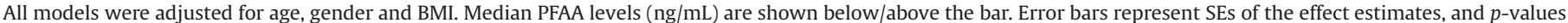
for trend are presented. 
Table 2

Risk of abnormal serum lipids (Logistic regression analysis) based on PFAA quartiles.

\begin{tabular}{|c|c|c|c|c|c|c|c|c|}
\hline \multirow[t]{2}{*}{ PFAAs (ng/mL) } & \multicolumn{2}{|l|}{$\mathrm{TC}$} & \multicolumn{2}{|l|}{ TG } & \multicolumn{2}{|l|}{ HDLC } & \multicolumn{2}{|l|}{ LDLC } \\
\hline & Crude OR & Adjusted $\mathrm{OR}^{\mathrm{a}}(95 \% \mathrm{CI})$ & Crude OR & Adjusted $\mathrm{OR}^{\mathrm{a}}(95 \% \mathrm{CI})$ & Crude OR & Adjusted $\mathrm{OR}^{\mathrm{a}}(95 \% \mathrm{CI})$ & Crude OR & Adjusted $\mathrm{OR}^{\mathrm{a}}(95 \% \mathrm{CI})$ \\
\hline \multicolumn{9}{|l|}{ PFOA } \\
\hline Quartile 1 & $1.00^{*}$ & 1.00 & 1.00 & 1.00 & 1.00 & 1.00 & $1.00^{* * *}$ & 1.00 \\
\hline Quartile 2 & 1.20 & $0.82(0.14-4.81)$ & 1.37 & $1.73(0.57-5.21)$ & 0.45 & $0.35(0.57-2.16)$ & 0.96 & $0.55(0.11-2.82)$ \\
\hline Quartile 3 & 5.95 & $2.60(0.56-12.09)$ & 1.85 & $1.03(0.33-3.20)$ & 1.30 & $0.89(0.19-4.19)$ & 5.04 & $1.70(0.40-7.19)$ \\
\hline Quartile 4 & 2.80 & $0.55(0.09-3.31)$ & 2.37 & $1.97(0.59-6.55)$ & 1.00 & $0.67(0.13-3.51)$ & 2.74 & $0.71(0.14-3.49)$ \\
\hline \multicolumn{9}{|l|}{ PFNA } \\
\hline Quartile 1 & 1.00 & 1.00 & 1.00 & 1.00 & 1.00 & 1.00 & 1.00 & 1.00 \\
\hline Quartile 2 & 1.13 & $0.73(0.17-3.19)$ & 1.54 & $1.33(0.46-3.91)$ & 0.97 & $0.88(0.16-4.81)$ & 2.30 & $2.37(0.57-9.90)$ \\
\hline Quartile 3 & 1.00 & $0.74(0.17-3.22)$ & 3.08 & $3.45(1.09-10.90)$ & 1.79 & $1.47(0.31-7.01)$ & 1.62 & $1.25(0.29-5.31)$ \\
\hline Quartile 4 & 2.30 & $1.03(0.24-4.46)$ & 1.28 & $0.80(0.26-2.49)$ & 1.38 & $1.06(0.20-5.57)$ & 3.95 & $2.51(0.59-10.74)$ \\
\hline \multicolumn{9}{|l|}{ PFDA } \\
\hline Quartile 1 & 1.00 & 1.00 & 1.00 & 1.00 & 1.00 & 1.00 & 1.00 & 1.00 \\
\hline Quartile 2 & 1.00 & $1.34(0.31-5.79)$ & 0.48 & $0.43(0.15-1.28)$ & 1.00 & $0.88(0.16-4.89)$ & 0.74 & $0.66(0.15-2.80)$ \\
\hline Quartile 3 & 0.81 & $1.80(0.39-8.27)$ & 0.58 & $0.71(0.24-2.06)$ & 0.63 & $0.59(0.09-3.85)$ & 1.26 & $3.26(0.77-13.88)$ \\
\hline Quartile 4 & 2.94 & $3.84(0.87-16.95)$ & 0.69 & $0.51(0.17-1.58)$ & 2.70 & $2.21(0.49-10.07)$ & 2.44 & $2.17(0.52-9.04)$ \\
\hline \multicolumn{9}{|l|}{ PFUdA } \\
\hline Quartile 1 & 1.00 & 1.00 & 1.00 & 1.00 & 1.00 & 1.00 & 1.00 & 1.00 \\
\hline Quartile 2 & 1.72 & $0.84(0.17-4.12)$ & 1.52 & $0.85(0.28-2.56)$ & 0.75 & $0.69(0.16-3.03)$ & 2.46 & $1.39(0.33-5.81)$ \\
\hline Quartile 3 & 3.64 & $2.94(0.58-14.89)$ & 0.78 & $0.39(0.13-1.20)$ & 0.56 & $0.51(0.10-2.47)$ & 3.32 & $2.56(0.52-9.87)$ \\
\hline Quartile 4 & 3.64 & $3.70(0.76-18.03)$ & 1.13 & $0.74(0.25-2.21)$ & 0.56 & $0.54(0.11-2.57)$ & 3.75 & $4.16(0.96-18.00)$ \\
\hline \multicolumn{9}{|c|}{ 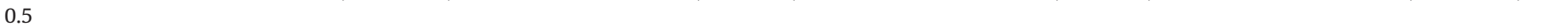 } \\
\hline \multicolumn{9}{|l|}{ PFOS } \\
\hline Quartile 1 & $1.00^{*}$ & 1.00 & 1.00 & 1.00 & 1.00 & 1.00 & $1.00^{*}$ & 1.00 \\
\hline Quartile 2 & 1.45 & $0.57(0.12-2.81)$ & 0.95 & $0.52(0.17-1.56)$ & 0.44 & $0.35(0.08-1.67)$ & 2.15 & $1.06(0.25-4.53)$ \\
\hline Quartile 3 & 2.44 & $0.82(0.17-3.91)$ & 1.63 & $0.93(0.31-2.80)$ & 0.45 & $0.36(0.08-1.72)$ & 2.57 & $1.11(0.25-4.82)$ \\
\hline Quartile 4 & 5.95 & $2.27(0.47-10.92)$ & 2.38 & $1.26(0.41-3.90)$ & 0.45 & $0.29(0.06-1.50)$ & 5.40 & $2.27(0.50-10.37)$ \\
\hline
\end{tabular}

\footnotetext{
a Adjusted for age, gender and BMI.

$* p<0.05$.

*** $p<0.01$.
}

level observed a modest but statistically significant, positive relationship between serum PFOA and TC, LDLC, for each increase in $1 \mathrm{ng} / \mathrm{mL}$ in serum PFOA, TC and LDLC increases by $0.10 \times 10^{-3}$ and $0.07 \times 10^{-3} \mathrm{mmol} / \mathrm{L}$, respectively (Sakr et al., 2007a). In a longitudinal study, Sakr et al. (2007b) found serum PFOA was positively associated with TC, with an increase of $0.03 \times 10^{-3} \mathrm{mmol} / \mathrm{L}$ of cholesterol for each $1 \mathrm{ng} / \mathrm{mL}$ increase in PFOA. Another longitudinal study investigated the association between within-individual changes in serum PFAAs and changes in serum lipid levels; they found that there was a tendency for people with greater declines in serum PFOA to have greater LDLC decrease. In a Danish population, $0.11 \mathrm{mmol} / \mathrm{L}$ higher concentration of TC was found with elevated interquartile range of PFOA (Eriksen et al., 2013). One study in American children and adolescents found that between the first and fifth quintiles of PFOA, there was a 0.12 and $0.10 \mathrm{mmol} / \mathrm{L}$ increase of TC and LDLC, respectively (Frisbee et al., 2010a). Another study in the general U. S. population showed that people in the highest PFOA quartile had TC levels $0.25 \mathrm{mmol} / \mathrm{L}$ higher than those in the lowest quartile (Nelson et al., 2010). In contrast, Olsen et al. conducted a study among 506 fluorochemical production workers and showed no association between PFOA and TC or LDLC (Olsen and Zobel, 2007). Emmett et al. (2006) assessed PFOA concentrations among 371 residents living around a DuPont plant and found no association between PFOA and TC. Results from Canadian Health Measures Survey exhibited an association between PFOA and TC in unweighted models, but this association became insignificant after weighting for sampling strategy (Fisher et al., 2013). In this study, positive associations of PFOA with TC and LDLC were observed both in crude and adjusted models. Those in the highest quartile of PFOA exposure had $1 n-T C$ and $1 n-L D L C$ levels 0.24 and $0.33 \mathrm{mmol} / \mathrm{L}$ higher than those in the lowest quartile, respectively. However, increased odds ratio of abnormal TC and LDLC was associated with elevated PFOA levels in crude models but not in adjusted models.

Only limited studies have examined the association of PFNA with serum lipid levels. Nelson et al. (2010) reported that people in the highest PFOA quartile had TC levels $0.36 \mathrm{mmol} / \mathrm{L}$ higher than those in the lowest quartile. Lin et al. (2009) investigated the relationship between PFAAs and components of metabolic syndrome using NHANES data and found that increased serum PFNA concentrations are associated with a lower prevalence of HDLC below the criteria. Our results for PFNA were consistent with Nelson's study; people in the highest PFNA quartile had $\ln -\mathrm{TC}$ and ln-LDLC levels 0.25 and $0.33 \mathrm{mmol} / \mathrm{L}$ higher than those in the lowest quartile, respectively (Nelson et al., 2010). However, our results showed no significant association of PFNA with odds ratio of abnormal serum lipids both in crude and adjusted models.

Until now, no association of serum lipids with PFDA or PFUdA has been reported. In this study, we showed that PFDA was associated with TC and HDLC across all models, while PFUdA was associated with TC and LDLC only in crude models. Moreover, PFDA or PFUdA was found not to be associated with odds ratio of abnormal serum lipids.

Eriksen et al. (2013) reported a $0.12 \mathrm{mmol} / \mathrm{L}$ higher concentration of TC with elevated interquartile range of PFOS in a Danish population consisting of 753 individuals. In the C8 Health Project, a positive linear association of PFOS with TC and LDLC among adults has been reported; people in the highest PFOS decile had TC levels $0.29 \mathrm{mmol} / \mathrm{L}$ higher than those in the lowest decile (Steenland et al., 2009). Another study from the C8 Health Project showed that there were a 0.22 and $0.15 \mathrm{mmol} / \mathrm{L}$ increases in the adjusted mean levels of TC and LDLC between the first and fifth quintiles of PFOS in children and adolescents (Frisbee et al., 2010b). Olsen et al. (2003) examined PFOS concentrations among 
flurochemical production workers and reported positive associations of PFOS with TC and TG . Another study done by Olsen et al. (1999) reported a negative association of PFOS with HDLC in samples of 1995, but this was not observed in 1997. Nelson et al. (2010) investigated four serum lipids and only found one significant positive association between PFOS and TC, those in the highest quartile of PFOS exposure had TC levels $0.35 \mathrm{mmol} / \mathrm{L}$ higher than those in the lowest quartile. Fisher et al. (2013) reported a positive association between PFOS and TC in unweighted models. In the present study, we found that PFOS was positively associated with TC and LDLC in crude models but not in adjusted models. In addition, elevated PFOS levels were linked to increased odds ratio of abnormal TC and LDLC in crude models.

The positive associations are unexpected based on toxicological studies, and the magnitudes of effects are inconsistent, which suggest that the associations may have a biological rather than a causal basis. Butenhoff et al. (2012) tested the hypothesis that PFOS and PFOA distribute into serum lipoprotein fractions so that increases in serum lipoproteins would result in corresponding increases in serum concentrations of PFOS and PFOA. The experimental results suggested that maximally $9 \%$ of PFOS distributed to lipoprotein-containing fractions, while only $1 \%$ or less of PFOA was so distributed. These data do not support a strong role for plasma lipoprotein fractions in explaining the inconsistent dose-response associations. Fletcher et al. (2013) presented the first evidence from a human population that PFOA and PFOS were associated with alterations in the levels of several gene transcripts involved in cholesterol transport. Their data were consistent with exposure to PFOA or PFOS contributing to elevated cholesterol, which has the potential to explain some of the epidemiological observations that have been reported for these compounds. However, Vanden Heuvel and John P (2013) questioned that Fletcher et al. examined only a few genes involved with cholesterol transport and may indicate perhaps just the opposite conclusion. Membrane transport may also play an important role if cholesterol and PFAAs share common processes or are influenced by common factors. Bijland et al. (2011) observed that dietary PFOS increased expression of PFOS genes involved in fatty acid uptake and transport in $\mathrm{APOE}^{*}$ 3-Leiden.CETP mice. Watkins et al. (2013) found that among four PFAAs (PFOA, PFNA, PFHxS, and PFOS), only PFOS was associated with LINE-1 DNA methylation, which is known to regulate gene expression and may influence serum lipids (Nelson et al., 2011). These hypotheses require substantive mechanistic research associated with hypercholesterolemia in the epidemiology studies.

Several limitations existed in this study. First, it was impossible to establish a causal inference due to the cross-sectional nature of this study (Grimes and Schulz, 2002). Second, both PFAAs and serum lipids might be jointly affected by some other attributes, substances or mechanisms that caused the associations between them (Steenland et al., 2010). Third, after the information of age, gender and BMI was adjusted, it might be still possible that residual confounding persisted due to unknown factors (Fisher et al., 2013).

We did not have any information on cholesterol lowering medication. The effect of systematic underestimation of higher cholesterol levels, however, if non-differential with respect to exposure, would most likely be null. Steenland et al. (2009) conducted an analysis including all subjects regardless of whether they were taking cholesterol medication. The results showed that those taking cholesterol medication had a significantly lower PFOA, but the effect was very modest (a decrease of only $4 \%$ ), with statistical significance primarily a function of the large sample size (69030 subjects). They also found that there was no difference in PFOS levels between those taking and not taking medication. Sensitivity analyses of those on cholesterol lowering medication did not impact the effect estimates (Château-Degat et al., 2010; Lin et al., 2009; Sakr et al., 2007a, b). In addition, the removal of participants using cholesterol lowering medication may bias the results by removing the diseased participants and leaving those with untreated high cholesterol.

Despite these limitations, the present study had a number of strengths. The large age range of participants made it possible to discuss the potential association between PFAAs and serum lipids among the whole population. In addition, we examined PFNA, PFDA and PFUdA, which receive less scientific attention but appear important.

\section{Conclusions}

Significant positive associations between PFOA and TC, PFOA and LDLC, PFNA and TC, PFNA and LDLC, PFDA and TC, PFDA and HDLC were observed in a Chinese population. Although we did not show a causal association between PFAA exposure and serum lipid levels due to the cross-sectional nature, our results provided clues for future epidemiologic and toxicology research.

\section{Acknowledgment}

This study was supported by the National Natural Science Foundation of China under Grant nos.41371488 and 41071355, the International Scientific Cooperation Program with Grant no. 2012DFA91150, and the Key Project of the Chinese Academy of Sciences under Grant no. KZZD-EW-TZ-12. We would like to thank the editors and reviewers for their valuable comments and suggestions.

\section{Appendix A. Supporting information}

Supplementary data associated with this article can be found in the online version at http://dx.doi.org/10.1016/j.ecoenv.2014.04.039.

\section{References}

Bijland, S., Rensen, P.C.N., Pieterman, E.J., Maas, A.C.E., van der Hoorn, J.W., van Erk, M.J., Havekes, L.M., van Dijk, K.W., Chang, S.-C., Ehresman, D.J., Butenhoff, J.L., Princen, H.M.G., 2011. Perfluoroalkyl sulfonates cause alkyl chain lengthdependent hepatic steatosis and hypolipidemia mainly by impairing lipoprotein production in APOE*3-Leiden CETP mice. Toxicol. Sci.. 123, 290-303.

Bjork, J.A., Butenhoff, J.L., Wallace, K.B., 2011. Multiplicity of nuclear receptor activation by PFOA and PFOS in primary human and rodent hepatocytes. Toxicology 288, 8-17.

Burstyn, I., 2013. Commentary: country doctor versus epidemiologist: how uncertainty analysis can help see what is in plain view. Epidemiology 24, 577-579.

Butenhoff, J.L., Pieterman, E. Ehresman, D.J. Gorman, G.S., Olsen, G.W Chang S.-C. Princen, H.M.G., 2012. Distribution of perfluoro-octanesulfonate and perfluorooctanoate into human plasma lipoprotein fractions. Toxicol. Lett. 210, 360-365.

Calafat, A.M., Kuklenyik, Z. Reidy, J.A., Caudill, S.P., Tully, J.S., Needham, L.L., 2007. Serum concentrations of 11 polyfluoroalkyl compounds in the US population: data from the National Health and Nutrition Examination Survey (NHANES) 1999-2000. Environ. Sci. Technol. 41, 2237-2242.

Château-Degat, M.-L., Pereg, D., Dallaire, R., Ayotte, P., Dery, S., Dewailly, É., 2010. Effects of perfluoro-octanesulfonate exposure on plasma lipid levels in the Inuit population of Nunavik (Northern Quebec). Environ. Res. 110, 710-717.

Conder, J.M., Hoke, R.A., Wolf, W.d., Russell, M.H. Buck, R.C., 2008. Are PFCAs bioaccumulative? A critical review and comparison with regulatory criteria and persistent lipophilic compounds. Environ. Sci. Technol. 42, 995-1003.

DeWitt, J.C., Peden-Adams, M.M., Keller, J.M., Germolec, D.R., 2012. Immunotoxicity of perfluorinated compounds: recent developments. Toxicol. Pathol. 40, $300-311$.

Elcombe, C.R., Elcombe, B.M., Foster, J.R., Chang, S.-C., Ehresman, D.J., Butenhoff, J.L., 2012. Hepatocellular hypertrophy and cell proliferation in Sprague-Dawley rats from dietary exposure to potassium perfluoro-octanesulfonate results from increased expression of xenosensor nuclear receptors PPAR alpha and CAR/PXR. Toxicology 293, 16-29.

Elcombe, C.R., Elcombe, B.M., Foster, J.R., Farrar, D.G., Jung, R., Chang, S.-C., Kennedy, G.L., Butenhoff, J.L., 2010. Hepatocellular hypertrophy and cell proliferation in Sprague-Dawley rats following dietary exposure to ammonium perfluorooctanoate occurs through increased activation of the xenosensor nuclear receptors PPAR alpha and CAR/PXR. Arch. Toxicol. 84, 787-798. 
Emmett, E.A., Zhang, H., Shofer, F.S., Freeman, D., Rodway, N.V., Desai, C., Shaw, L.M., 2006. Community exposure to perfluoro-octanoate: relationships between serum levels and certain health parameters. J. Occup. Environ. Med./Am. Coll. Occup. Environ. Med. 48, 771.

Eriksen, K.T., Raaschou-Nielsen, O., McLaughlin, J.K., Lipworth, L., Tjonneland, A., Overvad, K., Sorensen, M., 2013. Association between plasma PFOA and PFOS Levels and total cholesterol in a middle-aged Danish population. PloS One, 8.

Fang, X., Zou, S., Zhao, Y., Cui, R., Zhang, W., Hu, J., Dai, J., 2012. Kupffer cells suppress perfluorononanoic acid-induced hepatic peroxisome proliferatoractivated receptor alpha expression by releasing cytokines. Arch. Toxicol. 86, 1515-1525.

Fisher, M., Arbuckle, T.E., Wade, M., Haines, D.A., 2013. Do perfluoroalkyl substances affect metabolic function and plasma lipids?-Analysis of the 2007-2009, Canadian Health Measures Survey (CHMS) Cycle 1. Environ. Res. 121, 95-103.

Fitz-Simon, N., Fletcher, T., Luster, M.I., Steenland, K., Calafat, A.M., Kato, K., Armstrong, B., 2013. Reductions in serum lipids with a 4-year decline in serum perfluoro-octanoic acid and perfluoro-octanesulfonic acid. Epidemiology 24, 569-576.

Fletcher, T., Galloway, T.S., Melzer, D., Holcroft, P., Cipelli, R., Pilling, L.C., Mondal, D., Luster, M., Harries, L.W., 2013. Associations between PFOA, PFOS and changes in the expression of genes involved in cholesterol metabolism in humans. Environ. Int. 57-58, 2-10.

Frisbee, S.J., Shankar, A., Knox, S.S., Steenland, K., Savitz, D.A., Fletcher, T., Ducatman, A.M., 2010a. Perfluoro-octanoic acid, Perfluoro-octanesulfonate, and serum lipids in children and adolescents. Arch. Pediatr. Adolesc. Med. 164, 860-869.

Frisbee, S.J., Shankar, A., Knox, S.S., Steenland, K., Savitz, D.A., Fletcher, T., Ducatman, A.M., 2010b. Perfluoro-octanoic acid, perfluoro-octanesulfonate, and serum lipids in children and adolescents: results from the C8 Health Project. Arch. Pediatr. Adolesc. Med. 164, 860.

Fromme, H., Midasch, O., Twardella, D., Angerer, J., Boehmer, S., Liebl, B., 2007. Occurrence of perfluorinated substances in an adult German population in southern Bavaria. Int. Arch. Occup. Environ. Health 80, 313-319.

Fromme, H., Tittlemier, S.A., Volkel, W., Wilhelm, M., Twardella, D., 2009. Perfluorinated compounds-exposure assessment for the general population in western countries. Int. J. Hyg. Environ. Health 212, 239-270.

Giesy, J.P., Kannan, K., 2001. Global distribution of perfluoro-octane sulfonate in wildlife. Environ. Sci. Technol. 35, 1339-1342.

Grimes, D.A., Schulz, K.F., 2002. Bias and causal associations in observational research. The Lancet 359, 248-252.

Guo, F., Zhong, Y., Wang, Y., Li, J., Zhang, J., Liu, J., Zhao, Y., Wu, Y., 2011. Perfluorinated compounds in human blood around Bohai Sea, China. Chemosphere $85,156-162$.

Haug, L.S., Thomsen, C., Bechert, G., 2009. Time trends and the influence of age and gender on serum concentrations of perfluorinated compounds in archived human samples. Environ. Sci. Technol. 43, 2131-2136.

Holzer, J., Midasch, O., Rauchfuss, K., Kraft, M., Reupert, R., Angerer, J., Kleeschulte, P., Marschall, N., Wilhelm, M., 2008. Biomonitoring of perfluorinated compounds in children and adults exposed to perfluoro-octanoate-contaminated drinking water. Environ. Health Perspect. 116, 651-657.

Kannan, K., Corsolini, S., Falandysz, J., Fillmann, G., Kumar, K.S., Loganathan, B.G., Mohd, M.A., Olivero, J., Van Wouwe, N., Yang, J.H., Aldous, K.M., 2004. Perfluorooctanesulfonate and related fluorochemicals in human blood from several countries. Environ. Sci. Technol. 38, 4489-4495.

Lau, C., Anitole, K., Hodes, C., Lai, D., Pfahles-Hutchens, A., Seed, J., 2007. Perfluoroalkyl acids: a review of monitoring and toxicological findings. Toxicol. Sci. 99, 366-394.

Lin, C.-Y., Wen, L.-L., Lin, L.-Y., Wen, T.-W., Lien, G.-W., Hsu, S.H.J., Chien, K.-L., Liao, C.-C., Sung, F.-C., Chen, P.-C., Su, T.-C., 2013. The associations between serum perfluorinated chemicals and thyroid function in adolescents and young adults. J. Hazard. Mater. 244-245, 637-644.

Lin, C.Y., Chen, P.C., Lin, Y.C., Lin, L.Y., 2009. Association among serum perfluoroalkyl chemicals, glucose homeostasis, and metabolic syndrome in adolescents and adults. Diab. Care 32, 702-707.

Maher, J.M., Aleksunes, L.M., Dieter, M.Z., Tanaka, Y., Peters, J.M., Manautou, J.E., Klaassen, C.D., 2008. Nrf2-and PPAR alpha-Mediated Regulation of Hepatic Mrp Transporters after exposure to perfluoro-octanoic acid and perfluorodecanoic acid. Toxicol. Sci. 106, 319-328.

Martin, J.W., Mabury, S.A., Solomon, K.R., Muir, D.C., 2003. Dietary accumulation of perfluorinated acids in juvenile rainbow trout (Oncorhynchus mykiss). Environ. Toxicol. Chem. 22, 189-195.

Martin, J.W., Whittle, D.M., Muir, D.C.G., Mabury, S.A., 2004. Perfluoroalkyl contaminants in a food web from lake Ontario. Environ. Sci. Technol. 38, 5379-5385.

Meesters, R.J.W., Schroder, H.F., 2004. Perfluorooctane sulfonate-a quite mobile anionic anthropogenic surfactant, ubiquitously found in the environment. Water Sci. Technol. 50, 235-242.
Nelson, H.H., Marsit, C.J., Kelsey, K.T., 2011. Global methylation in exposure biology and translational medical science. Environ. Health Perspect. 119, 1528-1533.

Nelson, J.W., Hatch, E.E., Webster, T.F., 2010. Exposure to polyfluoroalkyl chemicals and cholesterol, body weight, and insulin resistance in the general US population. Environ. Health Persp. 118, 197-202.

Olsen, G.W., Burris, J.M., Burlew, M.M., Mandel, J.H., 2003. Epidemiologic assessment of worker serum perfluoro-octanesulfonate (PFOS) and perfluorooctanoate (PFOA) concentrations and medical surveillance examinations. J. Occup. Environ. Med. 45, 260-270.

Olsen, G.W., Burris, J.M., Ehresman, D.J., Froehlich, J.W., Seacat, A.M., Butenhoff, J.L., Zobel, L.R., 2007. Half-life of serum elimination of perfluoro-octanesulfonate, perfluorohexanesulfonate, and perfluoro-octanoate in retired fluorochemical production workers. Environ. Health Perspect. 115, 1298-1305.

Olsen, G.W., Burris, J.M., Mandel, J.H., Zobel, L.R., 1999. Serum perfluoro-octane sulfonate and hepatic and lipid clinical chemistry tests in fluorochemical production employees. J. Occup. Environ. Med. 41, 799-806.

Olsen, G.W., Butenhoff, J.L., Zobel, L.R., 2009. Perfluoroalkyl chemicals and human fetal development: an epidemiologic review with clinical and toxicological perspectives. Reprod. Toxicol. 27, 212-230.

Olsen, G.W., Ehresman, D.J., Buehrer, B.D., Gibson, B.A., Butenhoff, J.L., Zobel, L.R., 2012. Longitudinal assessment of lipid and hepatic clinical parameters in workers involved with the demolition of perfluoroalkyl manufacturing facilities. J. Occup. Environ. Med. 54, 974-983.

Olsen, G.W., Zobel, L.R., 2007. Assessment of lipid, hepatic, and thyroid parameters with serum perfluoro-octanoate (PFOA) concentrations in fluorochemical production workers. Int. Arch. Occ. Environ. Health 81, 231-246.

Pan, Y., Shi, Y., Wang, J., Cai, Y., Wu, Y., 2010. Concentrations of perfluorinated compounds in human blood from twelve cities in China. Environ. Toxicol. Chem. 29, 2695-2701.

Sakr, C.J., Kreckmann, K.H., Green, J.W., Gillies, P.J., Reynolds, J.L., Leonard, R.C., 2007a. Cross-sectional study of lipids and liver enzymes related to a serum biomarker of exposure (ammonium perfluoro-octanoate or APFO) as part of a general health survey in a cohort of occupationally exposed workers. J. Occup. Environ. Med. 49, 1086-1096.

Sakr, C.J., Leonard, R.C., Kreckmann, K.H., Slade, M.D., Cullen, M.R., 2007b. Longitudinal study of serum lipids and liver enzymes in workers with occupational exposure to ammonium perfluoro-octanoate. J. Occup. Environ. Med. 49, 872-879.

Seacat, A.M., Thomford, P.J., Hansen, K.J., Olsen, G.W., Case, M.T., Butenhoff, J.L., 2002. Subchronic toxicity studies on perfluoro-octanesulfonate potassium salt in cynomolgus monkeys. Toxicol. Sci. 68, 249-264.

Shoeib, M., Harner, T., Wilford, B.H., Jones, K.C., Zhu, J.P., 2005. Perfluorinated sulfonamides in indoor and outdoor air and indoor dust: occurrence, partitioning, and human exposure. Environ. Sci. Technol. 39, 6599-6606.

Skutlarek, D., Exner, M., Farber, H., 2006. Perfluorinated surfactants in surface and drinking water. Environ. Sci. Pollut. R 13, 299-307.

So, M.K., Yamashita, N., Taniyasu, S., Jiang, Q.T., Giesy, J.P., Chen, K., Lam, P.K.S., 2006. Health risks in infants associated with exposure to perfluorinated compounds in human breast milk from Zhoushan, China. Environ. Sci. Technol. 40, 2924-2929.

Steenland, K., Fletcher, T., Savitz, D.A., 2010. Epidemiologic evidence on the health effects of perfluoro-octanoic acid (PFOA). Environ. Health Perspect. 118, 1100-1108.

Steenland, K., Tinker, S., Frisbee, S., Ducatman, A., Vaccarino, V., 2009. Association of perfluoro-octanoic acid and perfluoro-octane sulfonate with serum lipids among adults living near a chemical plant. Am. J. Epidemiol. 170, 1268-1278.

Tittlemier, S.A., Pepper, K., Edwards, L., 2006. Concentrations of perfluorooctanesulfonamides in Canadian total diet study composite food samples collected between 1992 and 2004. J. Agric. Food Chem. 54, 8385-8389.

Vanden Heuvel and John P., 2013. Comment on Associations between PFOA, PFOS and changes in the expression of genes involved in cholesterol metabolism in humans by Fletcher et al., Environ. Int. 57-58 (2013) 2-10; Environ. Int. 61, $150-153$.

Watkins, D.J., Josson, J., Elston, B., Bartell, S.M., Shin, H.-M., Vieira, V.M., Savitz, D.A., Fletcher, T., Wellenius, G.A., 2013. Exposure to perfluoroalkyl acids and markers of kidney function among children and adolescents living near a chemical plant. Environ. Health Perspect. 121, 625-630.

Xiang, W., Du, J.B., 2009. Key points of the guidelines on prevention and treatment of blood lipid abnormality in chinese children and adolescents. Chin. J. Pediatr. 47, 637-639.

Zhao, S.P., 2008. Key points of the guidelines on prevention and treatment of blood lipid abnormality in Chinese adults. Pract. J. Clin. Med. 5, 3-6.

Zhang, T., Wu, Q., Sun, H.W., Zhang, X.Z., Yun, S.H., Kannan, K., 2010. Perfluorinated compounds in whole blood samples from infants, children, and adults in China. Environ. Sci. Technol. 44, 4341-4347. 